\title{
THz Radio Communication: Link Budget Analysis Towards 6G
}

\author{
Kari Rikkinen ${ }^{1}$, Pekka Kyösti ${ }^{2}$, Marko E. Leinonen ${ }^{1}$, Markus Berg ${ }^{3}$, Aarno Pärssinen ${ }^{1}$
}

\begin{abstract}
How well do upper millimeter-wave and terahertz frequency bands enable wireless communications? In this work, we approximate the current and estimate the future communication potential with emphasis on antenna and radio frequency hardware technologies, and radio propagation challenges. This is done by performing link budget evaluations with justified estimates of link budget calculus terms, such as the achievable or required noise figure, transmit power, and antenna gain. Estimates are based on current enabling technologies and needs to advance those. In RF viewpoint the bottlenecks are in generating sufficiently high transmit power and low noise with the support of very high antenna gains. As an example, we discuss opportunities around $300 \mathrm{GHz}$ frequency. Challenges to support $100 \mathrm{~Gb} / \mathrm{s}$ bit rate at $30 \mathrm{GHz}$ bandwidth on 10-meter link distance is analyzed for different kind of devices.
\end{abstract}

Index Terms - THz communication, link budget, link distance, $R F$ performance

\section{INTRODUCTION}

Trend in evolution of wireless communications systems is towards higher data rates, system capacities, system bandwidths, and operation frequencies. $5 \mathrm{G}$ is the first generation of mobile communication systems that supports millimeter wave band transmission for high speed wireless data transfer. It provides transmission rates on the order of several gigabits per second (Gbps) utilizing wide transmission bandwidths up to a few hundred megahertz $(\mathrm{MHz})$. The next generation, $6 \mathrm{G}$ wireless communication systems, are foreseen to expand operations to upper mm-wave (mmW) band (100-300 GHz) and terahertz (THz) band (300-3000 GHz) frequencies. Future 6G systems

\footnotetext{
${ }^{1}$ Kari Rikkinen, Marko E. Leinonen, Aarno Pärssinen are with the Centre for Wireless Communications, University of Oulu, 90014 Oulu, Finland (e-mails: kari.rikkinen@oulu.fi, marko.e.leinonen@oulu.fi, aarno.parssinen@oulu.fi).
}

will target to peak data rates up to terabit per second (Tbps) with low latency in transmission [1]. Significantly larger transmission bandwidths are needed compared to what $5 \mathrm{G}$ systems and spectrum allocations below $100 \mathrm{GHz}$ can provide.

International Telecommunication Union (ITU) and most national regulators have listed spectrum allocations up to $275 \mathrm{GHz}$. In ITU region 1, including Europe, a total of $81 \mathrm{GHz}$ spectrum is allocated for fixed/mobile communications in frequency range $100-275 \mathrm{GHz}$, with $23 \mathrm{GHz}$ of that allocation being in frequency range 252-275 $\mathrm{GHz}$ [2]. World Radiocommunication Conference 2019 (WRC-19) identified $137 \mathrm{GHz}$ of spectrum in the band $275-450 \mathrm{GHz}$ for land mobile and fixed service without any specific conditions to protect passive services, e.g., Earth explorationsatellite service (EESS), operating in $\mathrm{THz}$ frequencies [3]. Thus, a total of $160 \mathrm{GHz}$ spectrum is available for fixed/mobile communications in frequency range $250-450 \mathrm{GHz}$.

A fixed point-to-point link or an indoor system operating in upper $\mathrm{mmW}$ or terahertz band assumes usually line-of-sight (LOS) connection between transmitter and receiver. Attenuation of the radio signal in atmospheric propagation in LOS condition depends on free space path loss (Friis' formula), molecular absorption, and specific attenuation due to rain. Friis' transmission formula indicates that doubling the frequency quadruples the free space path loss that is due to reduced antenna element aperture as a function of frequency. In addition, molecular absorption spectral lines start impacting link budgets around $60 \mathrm{GHz}$ (oxygen) and above $300 \mathrm{GHz}$ (water). Rain attenuation is highly time and location dependent, but its impact must be included as a margin in long distance link designs.

Upper $\mathrm{mmW}$ and $\mathrm{THz}$ band antenna elements are typically tightly integrated with radio transceiver solution enabling the circuit or module level integration due to their small physical size. However, decreased antenna dimensions cause disadvantageous characteristics in the wave propagation. As the effective wave capturing area is smaller, the antenna element and thus received power reduces following the Friis' equation. THz antenna solutions must overcome this

\footnotetext{
${ }^{2}$ Pekka Kyösti is also with Keysight Technologies Finland Oy, 90590 Oulu, Finland (e-mail: pekka.kyosti@oulu.fi).

${ }^{3}$ Markus Berg is also with ExcellAnt Oy, 90590 Oulu, Finland (e-mail: markus.berg@oulu.fi).
} 
fundamental challenge by increasing the size of the antenna aperture using, e.g., lenses or phased arrays. In transmission and reception, large antenna aperture enables high directivity and gain to compensate free space propagation losses.

Convenient way to implement large size (and gain) antennas, e.g., for backhaul links is to use reflector-type dish antennas or dielectric collimating lens antennas. Lens is a specially shaped piece of dielectric material that focuses the field radiated by an antenna. Effectively a lens, as a component of antenna system, increases aperture of the antenna, providing higher gain and narrower antenna beam. Phased array as an alternative approach has been widely adopted at lower $\mathrm{mmW}$ region, e.g., $28 \mathrm{GHz}$ in 5G [4]. However, doubling the frequency quadruples the number of antennas for the same aperture. Moving from $28 \mathrm{GHz}$ to 200-300 GHz range a reasonable 32 element array scales up to the range of 2000 antennas and parallel transmit and receive RF paths. Such complexity is difficult to manage, and power consumption will limit strongly the practical implementation. Therefore, current $5 \mathrm{G}$ solutions are not easily scalable to $300 \mathrm{GHz}$ range.

Another fundamental physical constraint for $6 \mathrm{G}$ systems comes from the limited performance of electrical circuit technologies when approaching $\mathrm{THz}$ region. Radio transceiver solutions for current $4 \mathrm{G}$ and $5 \mathrm{G}$ mobile terminals are mainly implemented with complementary metal oxide semiconductor (CMOS) integrated circuit (IC) technology due to favorable cost, modularity and high level of integration. However, even today mobile terminal power amplifiers (PAs) are using either III-V technology, such as gallium arsenide (GaAs) or indium phosphide (InP), or silicon germanium (SiGe) heterojunction bipolar transistor (HBT) to overcome the limitation of the CMOS to generate enough radio frequency power efficiently at frequencies below $6 \mathrm{GHz}$. Also, the base station radios are utilizing other III-V technologies in the power amplifiers and low noise amplifiers (LNAs) to complement CMOS radio transceiver solutions to improve the radio performance. However, big and bulky discrete PA and LNA components are not anymore feasible solutions with small antennas even at lower $\mathrm{mmW}$ region. Highly integrated $\mathrm{CMOS}$ or $\mathrm{SiGe} \mathrm{HBT}$ $\mathrm{mmW}$ transceivers need to be adopted as IC solutions with integrated PAs and LNAs next to the antennas minimizing form factor and any RF loss degrading the performance in phased arrays. Future $6 \mathrm{G}$ frequencies at $100 \mathrm{GHz}$ and above will have also major challenge due to the available transistor speed (like $f_{\max }$ i.e. maximum frequency to achieve power gain) especially in silicon-based technologies such as CMOS and SiGe HBT. Approaching the boundary leads to exponential degradation in gain and output power as well as increased noise. Practical boundary to implement amplifiers is up to half of the $f_{\max }$. A recent example demonstrates $\mathrm{RF}$ transceiver operation up to $240 \mathrm{GHz}$ range using SiGe HBT technology with $\mathrm{f}_{\max } \approx 500 \mathrm{GHz}$ [5]. Even in this case performance is compromised compared to the capabilities of the same technology at frequencies below $100 \mathrm{GHz}$. In addition to thermal noise and output power limiting performance in absolute scale i.e. range, other non-idealities, like phase noise relative to signal level and bandwidth will have impact on applicable modulations by limiting maximum achievable signal-to-noise ratio (SNR). This is also highly dependent on applicable waveform, e.g., single carrier vs. orthogonal frequency division multiplexing (OFDM).

This paper discusses on key technology options in highly abstracted manner but keeping the most essential performance bottlenecks included. These are based on state-of-the-art technology boundaries in anticipated 6G link scenarios. Only a few early prototypes exist for short range links capable of operating at data rates close to 100 Gbps. New technologies may not quickly fill the gap between preferred and realistic performances. Therefore, it is of essence to study critical performance boundaries and build bridge from technologies to realistic communications concepts. For example, link budget analysis of fixed wireless links in [6] concludes that high bit rate connection over $1 \mathrm{~km}$ distance is possible but requires very high gains for the transmit and receive antennas. Is this scenario realistic to scale up for mass markets and what are technologies that can make it? These are key questions for $6 \mathrm{G}$ community in coming years. To boost the technology advancement, IEEE 802.15.3d has specified a physical layer for High Data Rate Wireless Multi-Media Networks operating in frequency range $252-325 \mathrm{GHz}$ with eight different channel bandwidths from $2.16 \mathrm{GHz}$ up to 69.12 GHz [7].

In this paper, we focus in $300 \mathrm{GHz}$ band operation in the link budget analysis and extend the consideration into small form factor devices. Carrier frequencies up to $300 \mathrm{GHz}$ are potential operation frequencies for the first $6 \mathrm{G}$ systems in $\mathrm{THz}$ band. In the following sections, we will consider various aspects in link budget analysis for $\mathrm{THz}$ band communication systems. Practical antenna and radio frequency hardware capabilities for fixed outdoor and low mobility indoor communication systems operating at $300 \mathrm{GHz}$ frequency are discussed and appropriate values selected for the link budget calculations. The link performance is analyzed with respect to achievable link distance with target system bandwidth and bit rate.

\section{LINK BUDGET EVALUATION METHOD}

The major challenges on realistic link budget and sufficient range determination on upper $\mathrm{mmW}$ band is to consider present and future performance 
of key enabling technologies. A simple link budget evaluation model for the requirement analysis of $\mathrm{THz}$ communication systems is adopted. It is intended to assess potential link distances, system bandwidths, bit rates and a support for mobility with respect to $\mathrm{THz}$ band transceiver technology capabilities and physical characteristics, like free space loss and molecular absorption, of the propagation medium to give comprehensive view on challenges and opportunities. A radio communication system model showing key transceiver parameters which affect to achievable link distance is given in Fig. 1. Power amplifier (PA) and low noise amplifier (LNA) present here the performance of the whole RF transmitter and receiver, respectively while digital signal processing (DSP) abstracts the modem processing to a single SNR number for simplicity.

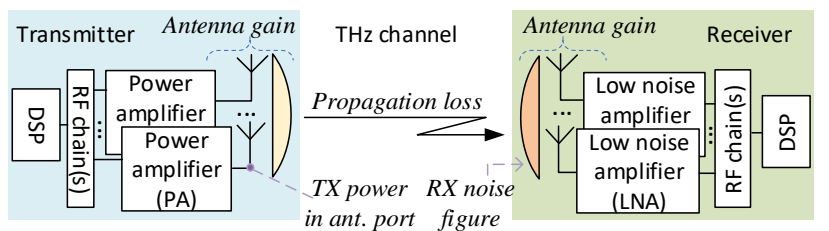

Figure 1. Radio communication system model

Inter-dependencies of various system parameters are illustrated in Fig. 2. A target bit rate can be realized with certain bandwidth and modulation/coding combinations. To match the target bit rate with the selected bandwidth we consider the single carrier modulation with M-ary phase-shift keying (M-PSK) or M-ary quadrature amplitude modulation (M-QAM) and a simple repetition code with selected code rate. The selected modulation/coding combination requires a certain system SNR, Target SNR, for reaching the bit rate target. This is compared to achievable SNR at the reception, $R x S N R$, which is specified by the noise power, propagation loss, antenna gains, and the transmit signal ( $T x$ ) power. The achievable Rx SNR includes all effects of transceiver RF implementation including data converters.

The maximum Tx power may be limited by regulations or by available component technologies. The former is often the case at lower frequencies and the latter at higher frequencies, where the capability to generate transmitted power and to achieve low noise are strictly bounded by speed of semiconductor technologies. Maximum frequency to achieve voltage or power gain i.e. $\mathrm{f}_{\mathrm{T}}$ or $\mathrm{f}_{\max }$ set strict physical limits for communication capabilities. Those are further lowered by parasitic effects in the semiconductors and interconnections between circuits. Here, in $T x$ power value, we include an estimate of achievable output for PA with selected technology examples and public analysis operating in upper $\mathrm{mmW}$ band. That is complemented with signal waveform dependent crest factor to model the backoff of the PA, and other RF transmitter chain impairments are included to a fixed 'post-PA' loss factor. The Rx $\mathrm{RF}$ chain impairments are included in the receiver noise figure (NF). System bandwidth, together with the NF, determine the total noise power level in the input of the Rx antenna.

The overall propagation loss is combination of the free space path loss, clutter loss, and atmospheric attenuation. These are all essentially dependent of the link distance. Furthermore, we assume that it is possible to arrange a direct LOS connection from transmitter to receiver. Transmitter and receiver antennas are modelled either with antenna element and lens or antenna array gains. The antenna gain affects also the beam width, which has impact on the supported mobility in highly directive communication systems.

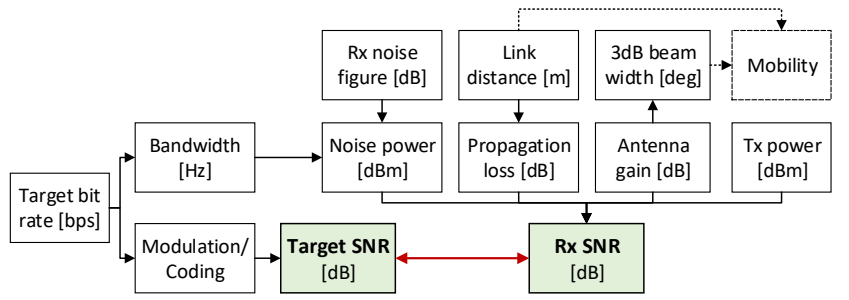

Figure 2. Inter-dependencies of selected RF system parameters.

\section{A. Target bit rate and bandwidth}

Potential use cases for $\mathrm{THz}$ band communications include wireless backhauling/fronthauling, close proximity communication systems, wireless links between servers inside a data center, and very high data rate connections needed for future $6 \mathrm{G}$ indoor services. Bit rate requirement may vary from 10 to 100 Gbps and even up to 1 Tbps with holographic type communications [1,7]. For the link budget calculations, we select primary link design parameters as follows: carrier frequency is 300 $\mathrm{GHz}$, system bandwidth $30 \mathrm{GHz}$, and target bit rate $100 \mathrm{Gbps}$. As the outcome of the analysis, we will determine the achievable link distance with given parameters. LOS model is mostly used as other scenarios lead easily to highly limited link ranges that are impractical in the field. However, for other channel non-idealities like beam misalignment or reflections a parameter describing link margin could be adopted to evaluate more challenging propagation scenarios. Unfortunately, based on the analysis it will be shown later that when extremely high data rates, bandwidths and carrier frequencies needed simultaneously the link budget will not allow similar margins that are anticipated in current cellular or local area communications below 6 $\mathrm{GHz}$ or not even below $100 \mathrm{GHz}$.

\section{B. Transmit power and noise figure}

One of the most critical parameters for the $\mathrm{THz}$ radio performance is the available RF power from 
the PA. Upper THz frequencies are using photonic based technologies to generate RF power while lower frequencies are relying on silicon or III-V based technologies such as gallium nitride $(\mathrm{GaN})$ or InP that enable small size PA development of $\mathrm{THz}$ frequency base station and mobile terminals. For compact, cost efficient and large-scale integration of $\mathrm{mmW}$ transceivers silicon-based technologies (SiGe HBT, CMOS or CMOS silicon on insulator (SOI)) are the viable solutions especially for mass markets.

A survey for different semiconductor technologies to generate RF power over wide range of frequencies is conducted in [8] indicating a strong dependence between the achievable saturated output power, operation frequency, and used semiconductor technology. As a summary, the achievable saturated radio frequency power decreases when operational frequency increases regardless of the used IC technology due to transistor speed limitations. For the link budget analysis, we choose two presentative values for the maximum saturated power of the PA, namely $+5 \mathrm{dBm}$ and $+13 \mathrm{dBm}$. The first value represents the state-of-the-art performance based on $\mathrm{SiGe}$ HBT and CMOS PAs at $200-250 \mathrm{GHz}$ based on reported values in $[5,8]$, while the latter value is for PA solution based on III-V technology at 300 $\mathrm{GHz}$ as reported in [8].

Also, noise figure of the receiver, is a technology dependent parameter that is a function of frequency and $\mathrm{f}_{\mathrm{T}}$ [9]. The reported noise figures of upper $\mathrm{mmW}$ band receivers or LNAs are in the range of $10-15 \mathrm{~dB}$ and the noise performance is limited by the used IC technology $[5,10]$. These $\mathrm{NF}$ values are higher than NFs of current $5 \mathrm{G}$ receivers due to carrier frequency. We selected three $\mathrm{NF}$ values $10 \mathrm{~dB}, 12 \mathrm{~dB}$ and $15 \mathrm{~dB}$ for our analyses to present expected NF performances of different kind of $\mathrm{THz}$ receivers. The two first values are for III-V technologies for larger devices considering the best LNA and leaving some margin for the contributions elsewhere in the receiver. However, tightly integrated, silicon based phased arrays can't be expected to achieve better than $15 \mathrm{~dB}$ noise figure

\section{Antenna gain}

In general, the only way to achieve high gain $\mathrm{THz}$ antenna is to increase the antenna aperture. Cassegrain reflector antenna has a large aperture and high gain $(55 \mathrm{dBi})$ but is not cost-effective or easily integrated [11]. State-of-the-art solution at $300 \mathrm{GHz}$ is to integrate dielectric lens to the system package, which typically means an antenna arrangement of one fixed beam. Practical gain values depend on the size, shape, and the dielectric properties of the lens, and are in the order of $20-$ $30 \mathrm{dBi}$ [12]. Switched beam antennas at lower $\mathrm{mmW}$ bands $(75 \mathrm{GHz})$ are achieved by integrating several antenna elements to a single lens. At mmW bands, phased antenna arrays have been found feasible in commercial $5 \mathrm{G}$ solutions.

When considering feasible antenna system for $\mathrm{THz}$ communication, the challenge is related to the beam steering capability. Lens antennas are fixed beam solutions by nature, which means that only one transceiver contributes to the communication to a single direction. This limitation is valid for a switched beam antenna in case of multiple antennas and transceivers integrated to a single lens. Further, the shape of one radiation beam is fixed, and thus geometrical coverage of one antenna element in high gain lens antenna is limited. Alternatively, large coverage is possible by using several antenna elements and beams simultaneously. Advantages of integrated lens antennas include efficient utilization of whole antenna aperture, stable radiation pattern over broad operation band and cost-effectiveness.

Phased antenna array has better beam steering capability. Practically, one active phased array can generate multiple simultaneous beams but leads to more complex RF front-end implementation. Challenges might arise from the element spacing when antennas are smaller than other integrated circuit components. Also, hundreds or thousands antenna elements require substantial number of transceiver chains which can increase implementation costs and will lead to challenges in the power consumption and thus also temperature control.

The proper antenna strategy for $\mathrm{THz}$ communication depends on the beam steering requirements. For a fixed link, the integrated lens antenna is a convenient solution. Switched beam lens antenna enables one or several simultaneous beams and could be optimum for low mobility applications. Phased arrays might show their advantage in case of moderate gain and high mobility communication applications. Trade-off between gain and mobility may lead to antenna system solutions including a combination of different basic high-gain antenna types.

For the link budget analysis, we select three type of antennas. Small lens antenna is realistic for local, low mobility indoor devices and large Cassegrain antenna for backhaul links. Mobile device and base station equipped with antenna arrays are included in the analysis to evaluate mobility aspects. Antenna gains 23-26 dBi represent calculated gains for small lens antennas with a diameter of $8-11 \mathrm{~mm}$ operating at 300 $\mathrm{GHz}$. Antenna gain $55 \mathrm{dBi}$ is a reported value for Cassegrain antenna operating at $275 \mathrm{GHz}$ [11]. Gain for the antenna array depends on array size, element gain and efficiency and is calculated as $17.1 \mathrm{dBi}$ and $29.1 \mathrm{dBi}$ for the 32 and 512 element arrays in mobile device and base station, respectively. 


\section{RESULTS}

Link budget calculations are conducted for short ranges up to $\sim 10 \mathrm{~m}$ using two different symmetric and static parameter sets for links (Links 1 and 2) and one mobile scenario (Link 3) including asymmetry in uplink and downlink directions. In addition, one fixed backhaul scenario for long range is calculated in Table I. Link 1 has small form factor devices at both ends such as access point or small portable device with a small lens antenna and silicon-based solution in the transceiver. Link 2 has small base station, front haul or laptop kind of devices with lens antennas and III-V technologies adopted in the transceiver to achieve better performance. Link 3 represents conventional mobile use scenario with asymmetric link consisting of mobile equipment implemented using silicon-based technologies while a small base station has better performance assuming adoption of III-V components as PAs and LNAs for performance and a large phased array. The base station is probably the most complex and challenging scenario for implementation as realizing poorly scalable III-V components as part of a 512-element array might not be practical. At least that scenario is significantly more complex and requires exceptional advances in mass market capable technologies with shorter range compared to stateof-the-art $5 \mathrm{G}$ mmW links. In backhaul scenario, it is possible to use the best performing technologies as antenna provides huge gain and link is fixed. These four link scenarios address comprehensively key reasons on the technology impact with associated cost and complexity for links ranging from centimeters up to kilometers.

Finally, also phase noise should be considered as it is often dominant factor especially in highorder modulations requiring SNR of $30 \mathrm{~dB}$ or even more. Here, waveform selection makes a big difference. In OFDM based systems phase noise is multiplied around each sub-carrier in down and up conversion, spreading the oscillator power all over the band. However, in single carrier modulations phase noise is strongly attenuated in phase locked loops (PLL) around RF oscillators and therefore filtering impact will relax the requirement. Unfortunately, due to transistor speed limitations multiplication of the lower frequency PLL signal is the only option to up or down convert the signal leading to higher noise. In addition, multiplication of wideband noise from PLL, that is typically at much lower level than close-in phase noise but still significant, will also migrate on the wideband channel [13]. This is partially unsolved issue. Therefore, both clock and PLL quality and proper waveform selection require careful attention in research towards $6 \mathrm{G}$.

Target SNR to Rx SNR comparison in Table I indicates that the target link distance of $10 \mathrm{~m}$ is achievable in link scenarios 2 and 3 but not with
1. In link 3 scenario, the achievable link distance is asymmetric in favor of downlink direction mainly due to larger amount of PAs in the base station transmitter. The target link distance of $1000 \mathrm{~m}$ for backhaul link can be well achieved with the determined transceiver parameters. Note, that parameter PA output power is saturated Tx power in Table I. Effective isotropic radiated power (EIRP) takes into account antenna element, lens and array gains and indicates the total power transmitted towards the main lobe. Modulation dependent back-off factor and post-PA loss, in total estimated as $3.6 \mathrm{~dB}$ for 16-QAM signal, are included in EIRP, as well.

Table I: Link budget calculation

\begin{tabular}{|c|c|c|c|c|c|c|}
\hline Parameter & Unit & Link 1 & Link 2 & Link 3-UL & Link 3- DL & Backhaul \\
\hline Frequency & $\mathrm{GHz}$ & \multicolumn{5}{|c|}{300} \\
\hline Wavelength & $\mathrm{m}$ & \multicolumn{5}{|c|}{$1.0 \mathrm{E}-3$} \\
\hline Link distance & $\mathrm{m}$ & \multicolumn{4}{|c|}{10} & 1000 \\
\hline Signal bandwidth & $\mathrm{GHz}$ & \multicolumn{5}{|c|}{30} \\
\hline Target bit rate & Gbps & \multicolumn{5}{|c|}{100} \\
\hline Modulation order & b/sym & \multicolumn{5}{|c|}{4} \\
\hline Code rate & & \multicolumn{5}{|c|}{0.8} \\
\hline Target SNR & $d B$ & \multicolumn{5}{|c|}{16.7} \\
\hline Free space path loss & $d B$ & \multicolumn{4}{|c|}{102.0} & 142.0 \\
\hline Atm. attenuation & $\mathrm{dB}$ & \multicolumn{4}{|c|}{0.0} & 3.0 \\
\hline Tot. propagation loss & $d B$ & \multicolumn{4}{|c|}{102.0} & 145.0 \\
\hline Antenna type & & lens & lens & Ph. array & Ph. array & lens \\
\hline \# of TX antennas & & 1 & 1 & 32 & 512 & 1 \\
\hline \# of RX antennas & & 1 & 1 & 512 & 32 & 1 \\
\hline Antenna gain $\mathrm{Tx}$ & $\mathrm{dBi}$ & 23.0 & 26.0 & 17.1 & 29.1 & 55.0 \\
\hline Antenna gain $\mathbf{R x}$ & $\mathrm{dBi}$ & 23.0 & 26.0 & 29.1 & 17.1 & 55.0 \\
\hline$-3 \mathrm{~dB}$ beam width & deg & 14.4 & 10.2 & 28.5 & 7.1 & 0.4 \\
\hline PA output power & $\mathrm{dBm}$ & 5.0 & 13.0 & 5.0 & 13.0 & 13.0 \\
\hline EIRP of TX & $\mathrm{dBm}$ & 24.4 & 35.4 & 33.6 & 65.6 & 64.4 \\
\hline Rx power & $\mathrm{dBm}$ & -54.6 & -40.7 & -39.4 & -19.4 & -25.5 \\
\hline Noise power & $\mathrm{dBm}$ & \multicolumn{5}{|c|}{-69.2} \\
\hline Rx noise figure & $d B$ & 15 & 10 & 12 & 15 & 10 \\
\hline Total noise power & $\mathrm{dBm}$ & -54.2 & -59.2 & -57.2 & -54.2 & -59.2 \\
\hline Rx SNR & $d B$ & -0.5 & 18.5 & 17.7 & 34.8 & 33.7 \\
\hline Rx SNR-target SNR & $d B$ & -17.2 & 1.8 & 1.0 & 18.1 & 17.0 \\
\hline
\end{tabular}

To enhance the analysis, achievable link distances for all link scenarios with different target bit rates are shown in Fig. 3. Seven different M-PSK/MQAM waveforms $(M=4,8,16,32,64,128,256)$ are used to cover the bit rate range from zero to 240 Gbps with the fixed signal bandwidth of $30 \mathrm{GHz}$. Backhaul is evaluated both with and without 20 $\mathrm{mm} / \mathrm{h}$ rain. Heavy rain of $20 \mathrm{~mm} / \mathrm{h}$ rate induces attenuation of $10.7 \mathrm{~dB} / \mathrm{km}$ at $300 \mathrm{GHz}$ [14]. Evidently, outdoor links are vulnerable to weather conditions which raises a challenge in $\mathrm{THz}$ band system design. As a note, we have assumed LOS connection and a perfect alignment between transceivers. If we add, e.g., $6 \mathrm{~dB}$ link margin, the achievable range would reduce to half. The $6 \mathrm{~dB}$ margin could correspond to a reflection from a reasonably good reflecting surface or half power beam misalignment at both ends of the link. 


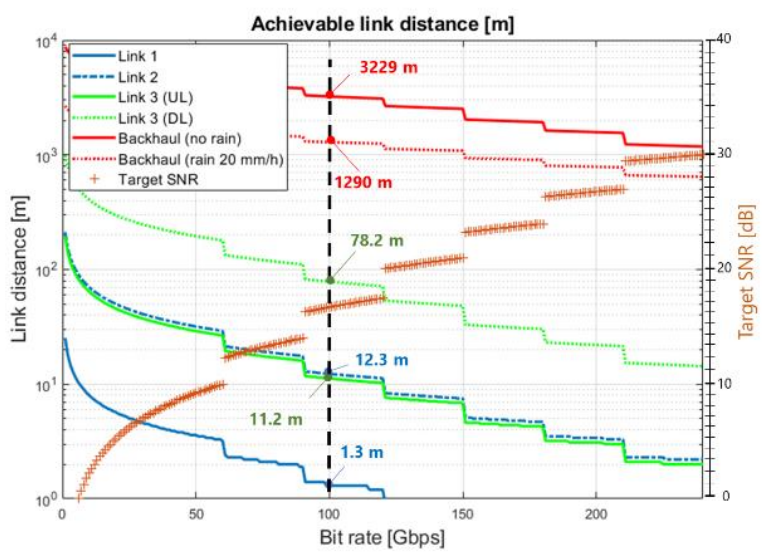

Figure 3. Achievable link distances and the target SNR at $300 \mathrm{GHz}$ carrier frequency

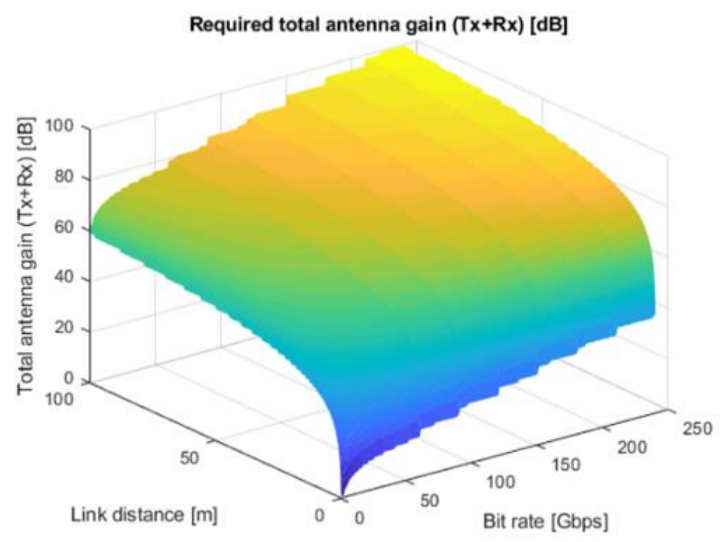

Figure 4. Required total antenna gain vs link distance with device targeted for link scenario 1

Figure 4 presents the total antenna gain $(\mathrm{Tx}+$ $\mathrm{Rx}$ ) requirement as a function of link distance and bit rate with the selected system parameters. Bit rate of $100 \mathrm{Gbps}$ is achievable with link distances $6.5 \mathrm{~m}$ and $61 \mathrm{~m}$, if the total antenna gain shared between transmitter and receiver is $60 \mathrm{dBi}$ and 80 $\mathrm{dBi}$, respectively.

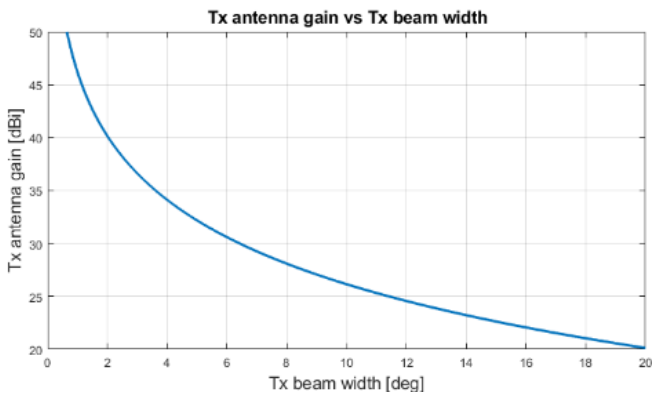

Figure 5. Half power beam width vs antenna gain

Antenna gain has an approximate relation to the half power $(-3 \mathrm{~dB})$ beam width. It is inversely proportional to the square-root-value of antenna gain (in linear unit). This approximation assumes rotationally symmetric antenna pattern. Figure 5 shows calculated half power beam width as a function of antenna gain. The support for mobility from beam tracking point of view can be speculated based on link distances and half power beam widths. For example, with link budget parametrization for Link 2 the Tx antenna gain is $26 \mathrm{dBi}$, corresponding to beam width of $10.2^{\circ}$. The half power area illuminated by such a beam is about $3.6 \mathrm{~m}$ wide at $10 \mathrm{~m}$ distance. Thus, a mobile transceiver would require new beam allocation approximately every $3.6 \mathrm{~m}$. With backhaul transceiver parameters the beam width is $0.4^{\circ}$, which would make beam alignment extremely difficult and mobility practically impossible.

\section{CONCLUSION}

We have evaluated communication link distances for links implemented with different technologies and complexity at $300 \mathrm{GHz}$ looking towards anticipated $6 \mathrm{G}$ use scenarios. Communications with high bit rates and bandwidths is feasible only for short range with transceivers having a small form factor including antennas. Achievable link distance with target bit rate of $100 \mathrm{Gbps}$ was limited to $1.3-12.3 \mathrm{~m}$ in such scenarios. Then with very large gain antennas in point-to-point outdoor links, distances can be extended significantly, i.e., above $3 \mathrm{~km}$ for backhaul applications. Furthermore, in long range outdoor links the impact of rain attenuation is considerable and heavy rain may halve the distance. Another challenge is implementation of phased arrays and misalignment of narrow pencil beams both in mobile and static scenarios.

The coverage is dominated by $\mathrm{Tx}$ power generation capability, receiver noise and used antenna gains at both link ends. Very high propagation loss inherent to $\mathrm{THz}$ frequencies must be compensated mainly by high gain antennas, since the RF power generation has a decreasing trend as function of frequency due to semiconductor technologies. Performance of transceivers using silicon technologies should approach current III-V state-of-the-art performance in performance, and architectural innovations. That may facilitate higher performance also in smaller devices and at lower cost.

Directivity of high gain antennas leads to narrow beam width. Even moderate mobility becomes challenging and would require both adaptive antenna solutions, like switched beam lens antennas, and sophisticated beam acquisition and tracking protocols. Long range links seem to be feasible only for fixed transceivers with very high antenna gains, since antennas are large and their beams are extremely narrow, e.g., $0.4^{\circ}$. However, narrow beams and high propagation losses relieve the network planning in the traditional interference handling point of view.

On the other hand, wide signal bandwidths introduce new interference scenarios. The RF carrier to carrier interference may not be critical 
problem, but legacy cellular systems at lower frequencies may influence the communication due to electromagnetic compatibility (EMC) reasons since, in our example, the baseband signal bandwidth goes up to $30 \mathrm{GHz}$. It is evident that transition from lower $\mathrm{mmW}$ region to $\mathrm{THz}$ will not come without need of major technological breakthroughs at different areas from electronics to algorithms. It is of utmost importance evaluate these opportunities holistically and realistically when concepting communications towards $6 \mathrm{G}$.

\section{ACKNOWLEDGMENT}

This work has been done in 6G Flagship programme funded by Academy of Finland (grant no. 318927 ).

\section{REFERENCES}

[1] M. Latva-aho, K. Leppänen," Key drivers and research challenges for $6 \mathrm{G}$ ubiquitous wireless intelligence", White Paper, Sep. 2019, https://www.6gchannel.com/, accessed Apr. 15, 2020.

[2] ECO Frequency Information System, https://www.efis.dk/, accessed Mar. 15, 2020.

[3] World Radiocommunication Conference 2019 (WRC-19), "Provisional Final Acts", Nov. 2019.

[4] X. Gu et al., "Development, Implementation, and Characterization of a 64-Element DualPolarized Phased-Array Antenna Module for 28-GHz High-Speed Data Communications," in IEEE Trans. on Microw. Theory and Techn., vol. 67, no. 7, pp. 2975-2984, July 2019.

[5] P. Rodriguez-Vazquez et al., "Signalprocessing Challenges in Leveraging 100 $\mathrm{Gb} / \mathrm{s}$ Wireless THz," in Proc. 2nd 6G Summit, Levi, Finland, Mar. 2020.

[6] T. Schneider et al.," Link Budget Analysis for Terahertz Fixed Wireless Links," IEEE Trans. on Terahertz Sci. and Techn., vol.2, no.2, pp. 250-256, Mar. 2012.

[7] T. Kürner, "Turning $\mathrm{THz}$ Communications into Reality: Status on Technology, Standardization and Regulation THz Communications," in Proc. 43 rd Int. Conf. on Infrared, Millimeter, and Terahertz Waves, Nagoya, Japan, Sep. 2018.

[8] H. Wang et al., "Power Amplifiers Performance Survey 2000-Present," https://gems.ece.gatech.edu/PA_survey.html, accessed Apr.15, 2020.

[9] S. Mattisson, "An Overview of 5G Requirements and Future Wireless Networks: Accommodating Scaling Technology," in IEEE Solid-State Circuits Magazine, vol. 10, no. 3, pp. 54-60, Aug. 2018.

[10] A. Tessmann et al., "A $300 \mathrm{GHz}$ low-noise amplifier S-MMIC for use in next-generation imaging and communication applications," 2017 IEEE MTT-S International Microwave Symposium (IMS), Honololu, HI, 2017, pp. 760-763.

[11] Terranova, "D5.3 - Report on final $\mathrm{THz}$ RF-frontend and antenna and optical RFfrontend for real-time", July 2019, https://ictterranova.eu/documents/deliverables/, accessed Apr. 15, 2020.

[12] N. Sarmah et al., "A Fully Integrated 240$\mathrm{GHz}$ Direct-Conversion Quadrature Transmitter and Receiver Chipset in SiGe Technology," IEEE Trans. on Microw. Theory and Techn., vol. 64, no. 2, pp. 562574, Feb. 2016.

[13] J. Chen et al., "Does LO Noise Floor Limit Performance in Multi-Gigabit MillimeterWave Communication?," in IEEE Microw. and Wireless Comp. Letters, vol.27, no.8, pp. 769-771, Aug. 2017.

[14] ITU-R, "Specific attenuation model for rain for use in prediction methods", Recommendation ITU-R P.838-3, Mar. 2005.

\section{BIOGRAPHIES}

Kari Rikkinen is currently a researcher in $6 \mathrm{G}$ Flagship programme with the Centre for Wireless Communications (CWC), University of Oulu. His current research activities include radio air interface physical layer research for $6 \mathrm{G}$ systems.

Pekka Kyösti is currently a senior specialist with Keysight Technologies Finland Oy, a research director in 6G Flagship programme and a docent with the Centre for Wireless Communications (CWC), University of Oulu. His present activities are channel modelling and over-the-air emulation for $5 \mathrm{G}$ systems, and radio channel characterization for $6 \mathrm{G}$ systems.

Marko E. Leinonen is currently a research manager with the Centre for Wireless Communications (CWC), University of Oulu. His research interests include wireless radio systems and quality topics in radio engineering.

Markus Berg is currently an Adjunct Professor with the Faculty of Information Technology and Electrical Engineering, University of Oulu. His current research interests include antennas and propagation for wireless communication, $\mathrm{THz}$ integrated antennas, wearable antennas, antennas for small devices, and GNSS reflectometry.

Aarno Pärssinen is with University of Oulu, Centre for Wireless Communications, Finland where he is currently a Professor leading Devices and Circuits research area in 6G flagship program. His research interests include wireless systems, ICs and transceiver architectures. He has authored one book, two book chapters, more than 100 international journal and conference papers and holds several patents. He served as a member of the technical program committee of Int. SolidState Circuits Conference in 2007-2017. 